\title{
Quantum Trajectories and Their Statistics for Remotely Entangled Quantum Bits
}

\author{
Areeya Chantasri, ${ }^{1,2}$ Mollie E. Kimchi-Schwartz, ${ }^{3}$ Nicolas Roch, ${ }^{4}$ Irfan Siddiqi, ${ }^{3}$ and Andrew N. Jordan ${ }^{1,2,5}$ \\ ${ }^{1}$ Department of Physics and Astronomy, University of Rochester, Rochester, New York 14627, USA \\ ${ }^{2}$ Center for Coherence and Quantum Optics, University of Rochester, Rochester, New York 14627, USA \\ ${ }^{3}$ Quantum Nanoelectronics Laboratory, Department of Physics, \\ University of California, Berkeley, California 94720, USA \\ ${ }^{4}$ Université Grenoble Alpes, Institut NEEL, F-38000 Grenoble, France \\ and CNRS, Institut NEEL, F-38000 Grenoble, France \\ ${ }^{5}$ Institute for Quantum Studies, Chapman University, 1 University Drive, Orange, California 92866, USA \\ (Received 31 March 2016; revised manuscript received 6 October 2016; published 14 December 2016) \\ We experimentally and theoretically investigate the quantum trajectories of jointly monitored transmon \\ qubits embedded in spatially separated microwave cavities. Using nearly quantum-noise-limited super- \\ conducting amplifiers and an optimized setup to reduce signal loss between cavities, we can efficiently \\ track measurement-induced entanglement generation as a continuous process for single realizations of the \\ experiment. The quantum trajectories of transmon qubits naturally split into low and high entanglement \\ classes. The distribution of concurrence is found at any given time, and we explore the dynamics of \\ entanglement creation in the state space. The distribution exhibits a sharp cutoff in the high concurrence \\ limit, defining a maximal concurrence boundary. The most-likely paths of the qubits' trajectories are also \\ investigated, resulting in three probable paths, gradually projecting the system to two even subspaces and \\ an odd subspace, conforming to a "half-parity" measurement. We also investigate the most-likely time for \\ the individual trajectories to reach their most entangled state, and we find that there are two solutions for the \\ local maximum, corresponding to the low and high entanglement routes. The theoretical predictions show \\ excellent agreement with the experimental entangled-qubit trajectory data.
}

DOI: 10.1103/PhysRevX.6.041052

\section{INTRODUCTION}

Measurement-induced entanglement of spatially separated quantum systems is a startling prediction of quantum mechanics [1-7]. Recent experiments have demonstrated this effect via single-photon heralding [8-11], as well as via continuous measurement of photons interacting with qubits [12]. The latter has the advantage of being able to investigate the physics of entanglement creation continuously, leading to new effects such as the sudden creation of entanglement after a finite measurement period, dubbed entanglement genesis [6]. However, many questions are outstanding, such as the following: (a) What is the complete characterization of the dynamics of entanglement creation as a continuous trajectory? (b) What is the statistical distribution of the entanglement at any time during the process? (c) What is the most-likely way entanglement is generated? In this work, we give a systematic answer to these questions, as well as others, by analyzing experimentally entangled quantum trajectories of jointly measured transmon qubits and showing excellent agreement with the theory developed here.

Published by the American Physical Society under the terms of the Creative Commons Attribution 3.0 License. Further distribution of this work must maintain attribution to the author(s) and the published article's title, journal citation, and DOI.
Subject Areas: Quantum Physics, Quantum Information

The rapid development of quantum information science in the superconducting domain [13] has seen an exponential increase in qubit coherence time within the past decade, leading to many scientific advances [14]. This technological progress has led to a wide variety of advances in quantum physics, as observed and controlled in these systems, including greater than $99 \%$ fidelity in single-qubit quantum gates [15], multiqubit-entanglement-generating gates [16], the violation of Bell's inequality [17], and quantum-process tomography [18]. Recent developments include the observation of quantum states of light in resonators [19], as well as nearly quantum-limited parametric amplifiers [20,21].

The improvement of coherent quantum hardware has brought with it a renewed focus on the physics of quantum measurement. Generalized measurements have been carried out in superconducting qubits [22], realizing probabilistic measurement reversal $[23,24]$, weak values [25,26], and their connection with generalized Leggett-Garg inequalities [27]. Continuous measurements [28] in superconducting systems have only recently been realized, owing to the challenge associated with high-fidelity detection of microwave signals near the single-photon level. In particular, experimental achievements include continuous feedback control $[29,30]$ and the tracking of trajectories in individual experiments in the plain measurement case [31-34] as well as with a concurrent Rabi drive [35]. 
These experiments show detailed quantitative agreement with theory, indicating a good understanding of quantities such as the most-likely path of the quantum state between boundary conditions, predicted with an action principle of an associated stochastic path integral [36-38].

Going beyond a single qubit opens the possibility of measurement-induced entanglement, using a dissipative process as a tool to generate quantum correlations. For quantum architectures building upon transitions at optical frequencies [8-11], this feat has typically been achieved by relying on the correlated detection of photons at the output of a beam splitter [1] to herald an entangled state. While powerful, this measurement protocol is binary and instantaneous, and it allows no insight into the dynamical processes underlying the generation of the entangled state. In solid-state systems, such as superconducting qubit transitions in the microwave regime, there has been tremendous interest in continuously generating bipartite [3-6,39] and multipartite [2,40,41] entangled states, using weak measurements that slowly interact with the qubits, in such a way that enables the resolution of the dynamical aspects of the entangling backaction. Analog feedback control can naturally be applied in the weak continuousmeasurement regimes [42-44], and digital feedbackgenerated entanglement has already been demonstrated [45]. Joint measurement is uniquely useful as a means to generate entanglement between remote qubits [12,46-50], for which no local coupling exists and therefore no unitary means of generating entanglement are available.

The chief advantage of the continuous approach is in the efficiency of entanglement generation. In contrast to photoncounting schemes (in which entanglement-generation rates are heavily limited by photon losses), continuous measurement enables an entanglement-generation rate limited only by the premeasurement initial state and the postselection criterion, which are both experimental choices. However, continuous measurements may be highly sensitive to dissipative losses and inefficiencies in amplification. Understanding and studying the dynamical processes underlying continuous measurement-induced entanglement is therefore critical for balancing these tradeoffs.

In this work, we combine an efficient quantum amplifier with a continuous half-parity measurement to conduct detailed experimental and theoretical investigations of the statistics of individual trajectories of qubit pairs as they undergo the entangling process. The half-parity measurement can distinguish between the even-parity and odd-parity subspaces, similar to a full-parity measurement, but also between the two states $|00\rangle$ and $|11\rangle$ in the even-parity subspace, leaving the odd subspace entangled. By peering into the ensemble, we can understand the full spectrum of evolution paths as the two-qubit state gradually projects onto the entangled subspace or onto a trivial separable state. We explore the probability distribution of the qubits' concurrence to understand how the distribution changes in time, from a separable state with zero concurrence, to projected states in either a separable subspace or an entangled subspace. This can also be seen from the most-likely path analysis, showing the emergence of different most probable paths for each final state. Moreover, we investigate the distribution of the time to maximum concurrence, finding that the most probable time to maximum values of concurrence has a bimodal structure. Studying the statistics of a large set of trajectories-rather than averaging over all of them to study the dynamics of the ensemble - enables an unprecedented understanding of the dynamics of entanglement creation under measurement.

The paper is organized as follows. In Sec. II, we describe our transmon qubit measurement setup and the method of reconstructing the joint trajectories. In Sec. III, we derive the concurrence-readout relation and compute the distribution of concurrence as the measurement backaction proceeds. This distribution is then compared with the data generated from the experiment. In Sec. IV, we turn to the most-likely path analysis, finding three possible paths that the joint system takes from a separable initial state to final entangled or separable states. The experimental most-likely paths are generated independently to compare with the theoretical prediction. We also discuss the distribution of the time for the two qubits to reach their maximum entanglement. The conclusions are presented in Sec. V. Additional details of the most-likely path calculations and a discussion of the parity meter are presented in the appendixes.

\section{TRAJECTORIES OF TRANSMON QUBITS}

We consider a two-qubit system realized by superconducting transmon qubits embedded in spatially separated microwave cavities in a setup optimized to reduce losses between the cavities. These qubits are jointly measured via a dispersive readout in a bounce-bounce geometry [see Fig. 1(a)] in which a microwave tone is sequentially reflected off of two copper cavities, each containing a transmon qubit, and subsequently amplified and measured via homodyne detection. The cavities are directly joined by a circulator that enforces the unidirectional transfer of the coherent state. A single transmon interacts with the light in its cavity via a dispersive interaction of the form

$$
H_{j}=\chi_{j} \sigma_{z}^{(j)} a^{\dagger} a,
$$

where $\chi_{j}$ is the dispersive interaction strength of the light interacting with the dipole moment of qubit $j$ (cross-Kerr nonlinearity between the qubit and the cavity), coupling to its $\sigma_{z}^{(j)}$ Pauli observable. The net effect of the bouncebounce geometry is that the effective interaction of the combined system is described by a Hamiltonian of the form $H_{1}+H_{2}$. After amplification and homodyne measurement, 

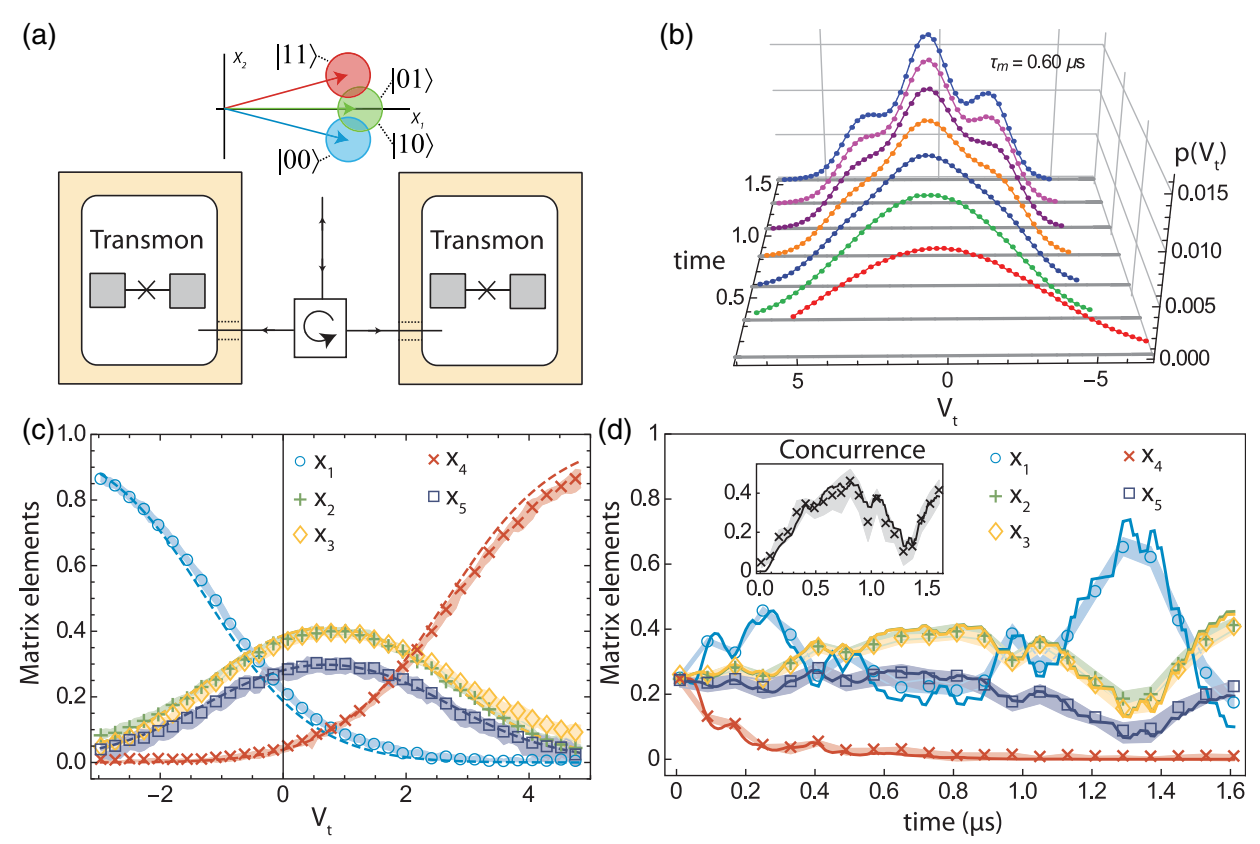

FIG. 1. Experimental setup and transmon qubit trajectories. Panel (a) shows a simplified illustration of the experimental setup. Two transmon qubits in two remote cavities are linked via a single microwave circulator in a bounce-bounce geometry; the output of the circulator is routed to a high-efficiency amplification chain (not shown). The upper inset represents the distribution of the microwave field in the $\left(X_{1}, X_{2}\right)$ quadrature plane at the output of the amplifier. The three possible outcomes correspond to three different subspaces: $|00\rangle,|11\rangle$, and the odd-parity subspace (spanned by the $|01\rangle$ and $|10\rangle$ states). Panel (b) shows the evolution in time of the measurement outcome probability distribution $p\left(V_{t}\right)$. Panel (c) shows the agreement between the experimentally generated conditional tomography $x_{i}\left(V_{t}\right)$ (symbols and shaded error bars) and the theoretical Bayesian reconstruction (dashed lines), for a single time $t=0.48 \mu$ s. In panel (d), we use such reconstructions to predict and verify the trajectory of a single iteration of the experiment, showing both density matrix elements $x_{i}$ and concurrence (inset).

this interaction gives a dispersive phase shift of $\pm \phi$ for states $|0\rangle,|1\rangle$. The shift $\phi$ is given, in general, by $\phi_{j}=\arg \left[\alpha_{|0\rangle, j}\right]-\arg \left[\alpha_{|1\rangle, j}\right]$, where $\alpha_{i, j}$ represents the intracavity coherent state conditioned on state $i$ of qubit $j$. In the fully symmetric, weak measurement case (when all qubit and cavity parameters are identical and $\left.\chi_{j=1,2} \ll \kappa\right)$, this phase shift is given by $\phi_{j} \approx 2 \chi_{j} / \kappa_{j}$, where $\kappa_{j}$ is the cavity damping rate of cavity $j$.

In the bounce-bounce geometry, there generally exists a probe frequency at which the dispersive shifts from both transmon qubits are the same $\left(\phi_{1}=\phi_{2}=\varphi\right)$, such that the measurement tone can acquire a phase shift of either $2 \varphi, 0$, $0,-2 \varphi$ for states $|00\rangle,|01\rangle,|10\rangle,|11\rangle$. For small $\chi / \kappa$, this results in a half-parity measurement on the two qubits, where the measurement result can distinguish between three subspaces: the $|00\rangle$ state, the $|11\rangle$ state, and the oddparity subspace, but not within the odd-parity subspace, spanned by the $|01\rangle$ and $|10\rangle$ states. Conditioning on the measurement results with a zero phase shift can lead to creation of an entangled state within the odd-parity subspace (superpositions of $|01\rangle$ and $|10\rangle$ ).

When the output of the second cavity [on the right in Fig. 1(a)] is directed to a nearly quantum-limited amplification chain, the instantaneous homodyne detection signal can be correlated with the measurement backaction on the qubits; therefore, it can be used to track the evolution of the system in time. By reducing the amplitude of the coherent state used to measure the system, we can engineer an entangling measurement with a characteristic measurement time ranging from several hundred nanoseconds to several microseconds: Critically, these time scales are easily resolvable experimentally. The dynamics or the trajectory of the system state can be obtained via the full master equations [46,47], using a two-cavity polaron transformation to account for the cavity degree of freedom, giving the stochastic master equation for the qubit trajectories. Alternatively, in a limit of large cavity decay rate $\kappa \gg|\chi|$, the qubit evolution can be continuously tracked via the quantum Bayesian approach [2,3], inferring the current states of the system from the measurement readouts and how likely they are to occur. The two approaches both show good agreement in tracking the qubit pair state [12].

In this paper, we focus on the quantum Bayesian approach, as it is directly related to the probability distribution of the measurement readout and naturally leads to the probability distribution of quantum trajectories. Let us denote $p\left(V_{t} \mid i\right)$ as a probability density function of a measurement readout $V_{t}$ conditioned on the two-qubit states $i$, where $i=1,2,3,4$ represent the states $|00\rangle$, $|01\rangle,|10\rangle,|11\rangle$, respectively. The quantum Bayesian update 
for this type of double-qubit measurement provides a convenient way to calculate the joint state at time $t$, given a known state at the initial time and the readout $V_{t}$,

$$
\rho_{i j}(t)=\frac{\rho_{i j}(0) \sqrt{p\left(V_{t} \mid i\right) p\left(V_{t} \mid j\right)} e^{-\gamma_{i j} t}}{\sum_{k=1}^{4} \rho_{k k}(0) p\left(V_{t} \mid k\right)},
$$

where $\rho_{i j}$ denotes the $i j$ element of the two-qubit density matrix and $\gamma_{i j}$ is a decoherence rate associated with the matrix element. The quantum Bayesian approach is equivalent to the positive-operator valued measure (POVM) formalism [51], provided we identify the measurement operators. In the case of no additional Rabi drives on either qubit, the measurement operators are given by

$$
\begin{aligned}
M_{V_{t}}= & \sqrt{p\left(V_{t} \mid \text { odd }\right)}(|01\rangle\langle 01|+| 10\rangle\langle 10|) \\
& +\sqrt{p\left(V_{t} \mid 1\right)}|00\rangle\left\langle 00\left|+\sqrt{p\left(V_{t} \mid 4\right)}\right| 11\right\rangle\langle 11|,
\end{aligned}
$$

where the distribution $p\left(V_{t} \mid\right.$ odd $)=p\left(V_{t} \mid 2\right)=p\left(V_{t} \mid 3\right)$ is the same for any state within the odd subspace. The POVM elements, $M_{V_{t}}^{\dagger} M_{V_{t}}$, correctly integrate to the identity operator in the two-qubit Hilbert space, understanding that we integrate with a measure for $V_{t}$. Applying the state update rule, conditioned on an observation of the readout $V_{t}, \rho(t)=M_{V_{t}} \rho(0) M_{V_{t}}^{\dagger} / \operatorname{Tr}\left[M_{V_{t}} \rho(0) M_{V_{t}}^{\dagger}\right]$ recovers Eq. (2) in the case of no extra decoherence $\gamma_{i j}=0$.

We define the readout $V_{t} \equiv(f / t) \int_{0}^{t} \tilde{V}\left(t^{\prime}\right) \mathrm{d} t^{\prime}-v_{0}$ as a time average of a raw homodyne voltage signal rescaled with a weight factor $f$ and an offset $v_{0}$, where $f$ is chosen so that the variance $\sigma_{V_{t}}^{2}=1 / 4 \eta_{m} t$ is a function of a quantum efficiency of the homodyne measurement, $\eta_{m} \approx 0.22$. The total probability distribution

$$
p\left(V_{t}\right)=\sum_{k=1}^{4} \rho_{k k}(0) p\left(V_{t} \mid k\right),
$$

shown in Fig. 1(b), slowly resolves into the three peaks expected for a half-parity measurement.

The conditional readout distributions are well approximated by Gaussian functions, giving $p\left(V_{t} \mid i\right)=$ $(t / \pi s)^{1 / 2} \exp \left\{-\left(V_{t}-\delta v_{i}\right)^{2} t / s\right\}$ with the centering signals $\delta v_{i}=v_{i}-v_{0}$ for $i=1, \ldots, 4$, where $s=1 / 2 \eta_{m}$. The measurement process cannot distinguish the two states in the odd-parity subspace; therefore, the readout distributions corresponding to the states $|01\rangle$ and $|10\rangle$ are completely (or nearly) overlapped, giving $\delta v_{2} \approx \delta v_{3} \approx 0$ and $-\delta v_{1} \approx \delta v_{4} \approx \delta v$. The measurement strength is characterized by an inverse of a characteristic measurement time $\tau_{m} \approx 1 / \delta v^{2} \eta_{m}$. The dephasing rates $\gamma_{i j}$ for $\delta v_{i} \neq \delta v_{j}$ are dominated by the effect of the distinguishability between states $i$ and $j, \gamma_{i j} \sim\left(\eta_{m}^{-1}-1\right)\left(\delta v_{i}-\delta v_{j}\right)^{2} / 4 s$ [2], resulting in the strong suppression of all off-diagonal elements except $\rho_{23}$. In an ideal half-parity measurement, the decay of $\rho_{23}$ would be limited only by the intrinsic lifetimes of the qubits; however, we must additionally account for experimental imperfections in the matching of $\delta v_{2}$ and $\delta v_{3}$ and for the loss of photons between the two cavities. These effects are included in the (slightly time-dependent) dephasing rate $\gamma_{23}$.

Since we expect most of the off-diagonal terms to damp quickly, we only consider five relevant density matrix elements: $x_{1} \equiv \rho_{11}, x_{2} \equiv \rho_{22}, x_{3}=\rho_{33}, x_{4} \equiv \rho_{44}$, and $x_{5} \equiv$ $\left|\rho_{23}\right|$ [52]. In order to compare the Bayesian prediction to the true density matrix, we perform conditional tomography $[12,53]$ to experimentally reconstruct the full experimental mapping $V_{t} \mapsto \rho\left(V_{t}, t\right)$. In conditional tomography, we collect an ensemble of trajectories that reach a particular $V_{t}$ at time $t$, and tomographically reconstruct that ensemble. This provides a density matrix conditioned on $V_{t}$. In Fig. 1(c), we show good agreement between the Bayesian prediction and conditional tomography reconstruction of $x_{i}\left(V_{t}\right)$ for a single measurement time $t=0.48 \mu \mathrm{s}$. We show examples of transmon qubit trajectories in Fig. 1(d) for an initial state prepared in a product of $\hat{x}$ states, i.e., the equal superposition state $(1 / 2)(|00\rangle+|01\rangle+|10\rangle+|11\rangle)$, such that the five relevant density matrix elements are $x_{1}^{0}=x_{2}^{0}=x_{3}^{0}=x_{4}^{0}=x_{5}^{0}=1 / 4$. We show both the Bayesian reconstruction and the tomographic verification, which are in good agreement. This verifies that the Bayesian reconstruction can be used to faithfully translate a noisy measurement signal into the stochastic evolution of a joint quantum state.

We conclude this section with a qualitative discussion of the mechanisms behind the entanglement dynamics, which will be followed up with a quantitative analysis in the next section. As discussed previously, the half-parity measurement can distinguish between the two evenparity states but not within the odd-parity subspace. We can think of this measurement as a continuous version of an ideal projective measurement with three projectors $\Pi_{1}=|00\rangle\left\langle 00\left|, \Pi_{\text {odd }}=\right| 01\right\rangle\langle 01|+| 10\rangle\left\langle 10\left|, \Pi_{3}=\right| 11\right\rangle\langle 11|$, which partition unity in the two-qubit-state space. Given an arbitrary separable pure state, $|\psi\rangle=\left(a_{1}|0\rangle_{1}+b_{1}|1\rangle_{1}\right) \times$ $\left(a_{2}|0\rangle_{2}+b_{2}|1\rangle_{2}\right)$, textbook wave-function collapse upon measurement of the odd result gives the (un-normalized) state $|\tilde{\psi}\rangle=a_{1} b_{2}|01\rangle+b_{1} a_{2}|10\rangle$. Clearly, for certain values of $a_{1}, b_{1}, a_{2}, b_{2}$, the postcollapse state will be entangled. In particular, the choice $a_{1}=b_{1}=a_{2}=b_{2}=$ $1 / \sqrt{2}$ previously mentioned will result in a maximally entangled state. Mathematically, entanglement is created because the odd projection operator cannot distinguish within the odd subspace.

Of the three possibilities between collapse to the two even states or to the odd subspace, quantum mechanics only tells us the statistics of these events happening; it does not predict which will occur in a given run. In addition, in the continuous measurement case, we can only predict the 
statistics of the system by choosing one eventual path among the various choices. However, because the probability distribution of the results is of the simple form (4), we may understand the gradual transition from separable to entangled as a given measurement result indicating which basis state the system is collapsing into, masked by additive quantum noise. Transitions from one branch to another can occur and may be understood as originating from the tails of the distributions overlapping. As time develops, those tails decrease, so a larger (and less likely) quantum fluctuation is required in order to cause the quantum system to transition from one branch to another. This increase of knowledge resulting in an altered quantum state may be understood as Bayesian inference, suitably generalized to take quantum entanglement into account, Eq. (2).

\section{CONCURRENCE TRAJECTORIES AND THEIR DISTRIBUTION}

We now give a quantitative analysis of the statistical process of entanglement creation for continuous measurement. As a measure of entanglement between two parties such as the transmon qubits, concurrence is a convenient choice and can be computed directly from the density matrix of the system [54]. The concurrence formula for the half-parity setup is greatly simplified because of the suppression of most matrix elements, resulting in an $\mathrm{X}$-shape density matrix, of which the concurrence is calculated from $[54,55]$

$$
\mathcal{C}(t)=2 \max \left\{0, x_{5}(t)-\sqrt{x_{1}(t) x_{4}(t)}\right\} .
$$

The value of concurrence ranges from 0 for a separable state to 1 for the Bell states. The concurrence trajectory of an exemplar trajectory is shown in the inset to Fig. 1(d). In this section, we use the simplified formula Eq. (5) to show that the concurrence of the two-qubit state can be determined directly from the measurement readout, which then leads to the derivation of the concurrence probability distribution as a function of the measurement readout and measuring time.

\section{A. Concurrence-readout relationship}

Let us consider the concurrence formula in Eq. (5), where its value is determined by the second term in the brackets, which we denote $c_{t} \equiv 2\left\{x_{5}(t)-\sqrt{x_{1}(t) x_{4}(t)}\right\}$. If $c_{t}$ is a non-negative quantity (i.e., $c_{t} \geq 0$ ), then the concurrence is simply given by $\mathcal{C}(t)=c_{t}$. At the end of this section, we show that this is always the case for our chosen initial qubit state and parameter regimes, but it is not true in general [6]. From the Bayesian update in Eq. (2), we calculate the quantity

$$
c_{t}=\frac{2}{N}\left\{x_{5}^{0} \sqrt{P_{2} P_{3}} e^{-\gamma t}-\sqrt{x_{1}^{0} x_{4}^{0} P_{1} P_{4}}\right\},
$$

where $x_{i}^{0}$ for $i=1, \ldots, 5$ are the matrix elements of the initial qubit state, and $\gamma=\gamma_{23}$. We have used simplified notations $P_{i} \equiv p\left(V_{t} \mid i\right)$ for the probability distributions, and $N$ for a normalized factor given by $N=\sum_{k=1}^{4} x_{k}(0) P_{k}$.

Substituting the probability distribution functions with the Gaussian functions of the means $\delta v_{i}$ for $i=1, \ldots, 4$, we obtain a form of $c_{t}$ explicitly as a function of $V_{t}$ and $t$,

$c_{t}\left(V_{t}, t\right)=\frac{2}{\mathcal{M}}\left\{x_{5}^{0} e^{\left(\alpha_{23} V_{t}-\beta_{23}-\gamma\right) t}-\sqrt{x_{1}^{0} x_{4}^{0}} e^{\left(\alpha_{14} V_{t}-\beta_{14}\right) t}\right\}$,

where the prefactor is given by $\mathcal{M}=\sum_{i=1}^{4} x_{i}^{0} e^{2 \alpha_{i} V_{t} t-2 \beta_{i} t}$ using a set of defined variables: $\alpha_{i}=\delta v_{i} / s, \alpha_{i j}=\alpha_{i}+\alpha_{j}$, $\beta_{i}=\delta v_{i}^{2} / 2 s, \beta_{i j}=\beta_{i}+\beta_{j}$, and $s=1 / 2 \eta_{m}$.

The quantity $c_{t}$ in Eq. (7) would represent the actual concurrence of the qubit state at any time $t$, if $c(t) \geq 0$ is satisfied. For our chosen initial state, a product of singlequbit $\hat{x}$ states, $x_{1}^{0}=x_{2}^{0}=x_{3}^{0}=x_{4}^{0}=x_{5}^{0}=1 / 4$, the quantity $c_{t}$ is non-negative whenever the condition $\left(\gamma-\alpha_{23} V_{t}+\right.$ $\left.\beta_{23}\right)<\left(\beta_{14}-\alpha_{14} V_{t}\right)$ is true. From the experimental data (e.g., for the setup with $\tau_{m}=0.60 \mu \mathrm{s}$ ), we have $-\delta v_{1} \approx \delta v_{4}$ and $\delta v_{2} \approx \delta v_{3} \approx 0$ (giving $\alpha_{14}, \quad \alpha_{23}, \quad \beta_{23} \approx 0$ ) and $\beta_{14} \sim 3.2 \mathrm{MHz}$, while $\gamma<0.6 \mathrm{MHz}$. Therefore, the condition is always satisfied, and the second term in the brackets of Eq. (7) decays faster than the first term, resulting in a quantity that is always non-negative. Consequently, the quantity in Eq. (7) gives the concurrence-readout relationship $\mathcal{C}\left(V_{t}, t\right)=c_{t}\left(V_{t}, t\right)$, and the concurrence at any time $t$ can be determined directly from the time-averaged measurement readout $V_{t}$.

The concurrence formula in Eq. (7) can be simplified further by considering a perfectly symmetric half-parity measurement, $\delta v_{2}=\delta v_{3}=0$ and $-\delta v_{1}=\delta v_{4}=\delta v$. Given the initial state, a product of two qubit $\hat{x}$ states, the concurrence is then given by

$$
\mathcal{C}_{\mathrm{ps}, \hat{x}}\left(V_{t}, t\right)=\frac{e^{-\gamma t}-e^{-\delta v^{2} t / s}}{1+\cosh \left(2 V_{t} \delta v t / s\right) e^{-\delta v^{2} t / s}},
$$

where the subscript "ps, $\hat{x}$ " indicates the perfectly symmetric half-parity measurement given the specific initial state.

\section{B. Probability density function for concurrence trajectories}

From the direct relationship between the measurement readout and the concurrence of the qubit state, the probability density function of the concurrence can be derived from the probability distribution of the time-averaged signal $V_{t}$. The distribution of the time-averaged readout is given by 


$$
p\left(V_{t}\right)=\sum_{i=1}^{4} x_{i}^{0} p\left(V_{t} \mid i\right)
$$

The variance of the distribution $\sigma_{V_{t}}^{2}=s / 2 t$ narrows as time increases, leading to the collapse of the joint qubit state into three categories: $|00\rangle$ state, $|11\rangle$ state, and some superposition state of $|01\rangle$ and $|10\rangle$ after a few characteristic measurement times $\tau_{m}$.

Knowing the probability density function of the timeaveraged signal, we follow the transformation of random variables $V_{t} \rightarrow \mathcal{C}$ using the concurrence-readout relationship, Eq. (7) [or Eq. (8) for a perfectly symmetric case]. The concurrence $\mathcal{C}\left(V_{t}, t\right)$ is not a monotonic function in $V_{t}$; instead, it has a bell-like shape, as shown in the inset of Fig. 2(a). We write the cumulative distribution function of the concurrence $F_{\mathcal{C}, t}(c)=p_{\mathcal{C}, t}(\mathcal{C} \leq c)=p\left(V_{t} \leq V_{-}\right)+$ $\left\{1-p\left(V_{t} \leq V_{+}\right)\right\}$, where $p_{\mathcal{C}, t}(c)$ is a probability density function for the concurrence, and $V_{+}, V_{-}$are two solutions that arise from solving Eq. (7) [or Eq. (8)], $\mathcal{C}\left(V_{t}, t\right)=c$. The concurrence distribution is then obtained by taking a derivative of the cumulative distribution,

$$
p_{\mathcal{C}, t}(c)=p\left(V_{-}\right)\left|\frac{\partial V_{-}}{\partial c}\right|+p\left(V_{+}\right)\left|\frac{\partial V_{+}}{\partial c}\right|,
$$

noting that $V_{-}(c, t)$ and $V_{+}(c, t)$ are functions of the concurrence $c$ and time $t$. The full solution of $p_{\mathcal{C}, t}(c)$ is quite lengthy and is not shown.

We show in Fig. 2(a) the plots of concurrence probability distributions (10) for different values of time $t$, and in Figs. 2(b) and 2(c) the density plot comparing the theory and the transmon experiment. At an early time, the distribution of concurrence is narrowly peaked near its maximum, which increases over time, whereas at later times, a second peak emerges near the zero concurrence, showing a bimodal distribution. In Fig. 2(b), the theoretical histogram for the concurrence is obtained by integrating the theory curves, Eq. (10), for the probability over small intervals $\delta c \approx 0.015$. This integration makes a fair comparison with the histogram of the experimental data in Fig. 2(c), calculated with a bin size of 0.015 . We note that a short delay in the experimental entanglement creation is a result of the cavity ring-up time, which will be discussed more in the next section.

We stress that the concurrence distribution has a sharp upper bound [shown as a grey dotted curve in Fig. 2(a)], which the concurrence cannot exceed. In order to understand why the probability distribution for the concurrence has a sharp upper cutoff at any time, we recall that the density matrix of the two-qubit system, conditioned on the time-integrated readout $V_{t}$, is entirely specified by that (random) outcome, together with the initial state, the dephasing rate, and other parameters of the problem, Eq. (2). Consequently, the concurrence is controlled by $V_{t}$, as in Eq. (8). As can be seen from the inset of Fig. 2(a),
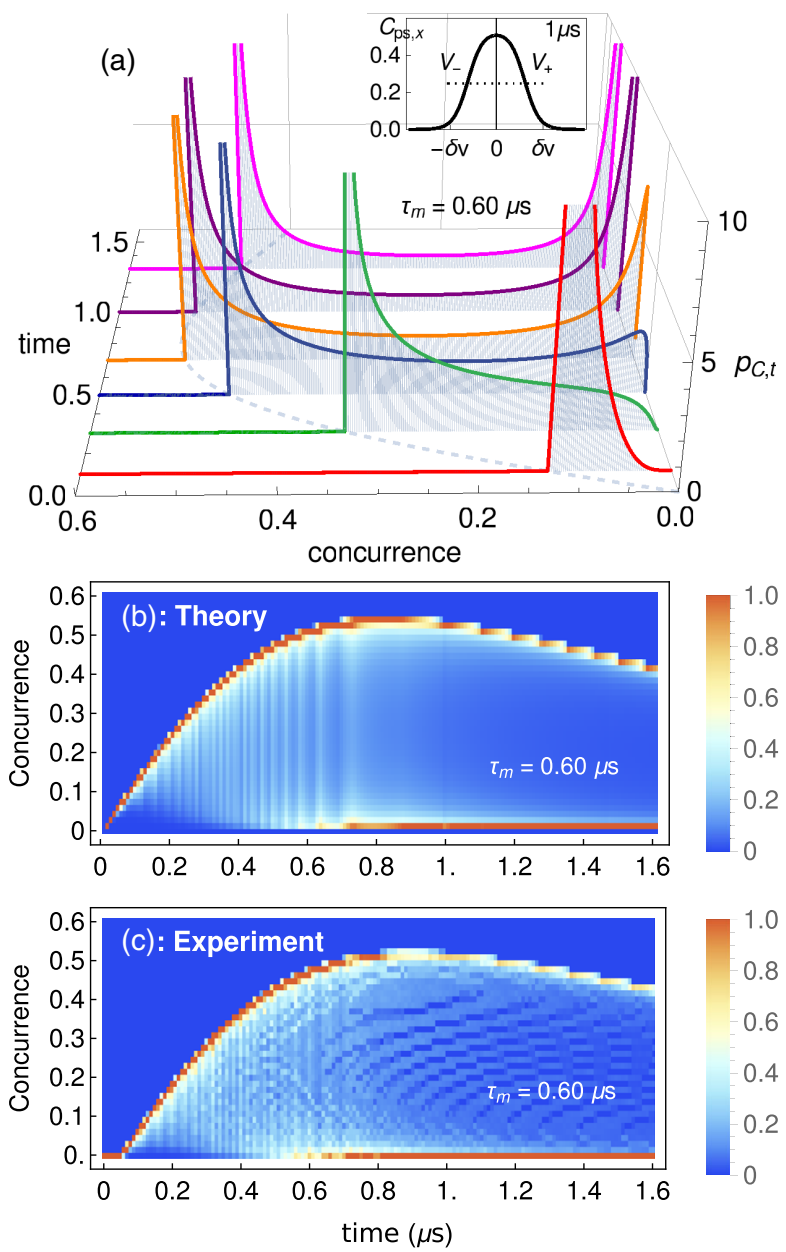

FIG. 2. Concurrence distribution for the qubits under the halfparity measurement. In panel (a) we plot the concurrence probability density function, Eq. (10), for different values of time. The values of time for the presented curves are chosen so as to see their unique features as they develop. The grey dotted curve joining the high-concurrence peaks shows the concurrence upper bound, Eq. (11). The inset shows an example of how the concurrence (at time $t=1 \mu \mathrm{s}$ ) varies as a function of the readout $V_{t}$. Panels (b) and (c) are the histograms of the concurrence at any time points from $t=0$ to $T=1.6 \mu \mathrm{s}$ (with a step size $0.01 \mu \mathrm{s}$ ), comparing theory and experimental data. For the theory plot, we coarse-grain the distribution $p_{\mathcal{C}, t}(c)$ in Eq. (10) by integrating it with a pixel size $\delta c \sim 0.015$, which is the bin size of the experimental histogram. For presentation purposes, a histogram at any time $t$ is normalized by its maximum element.

the concurrence, plotted as a function of the measured signal $V_{t}$, is bounded from above for any fixed time $t$ by some amount we call $\mathcal{C}_{\max }$, and consequently, any value of concurrence higher than that maximum (whose value will change as the time increases) cannot be realized. Therefore, the probability distribution of concurrence has a sharp upper cutoff given by $\mathcal{C}_{\max }(t)$. Physically, this indicates that there is an upper limit on how fast entanglement can be created by the continuous measurement in this situation, even for rare events of the measurement process. 
For the perfectly symmetric case in Eq. (8), we find an analytic solution for the upper bound of the concurrence, knowing that $\cosh (x)$ has its minimum at $x=0$ and $\mathcal{C}_{\mathrm{ps}, \hat{x}}\left(V_{t}\right)$ has its maximum at $V_{t}=0$; consequently, the concurrence upper bound is given by

$$
\mathcal{C}_{\max , \mathrm{ps}, \hat{x}}(t)=\frac{e^{-\gamma t}-e^{-\delta v^{2} t / s}}{1+e^{-\delta v^{2} t / s}} .
$$

The behavior of this bound is a result of two competing rates - the extra dephasing rate $\gamma$ and a measurement rate $\delta v^{2} / s$. Equation (11) increases from zero for small times and decays for a long time after reaching its maximum concurrence, as seen in Fig. 2. The maximum possible concurrence for this qubit half-parity measurement and the time this happens can be obtained from this relation. More about the time to reach maximum concurrence will be discussed in Sec. IV C.

\section{MOST-LIKELY PATH ANALYSIS}

In addition to the distribution analysis in the previous section, where we treated the concurrence at each point in time independent from any other times, we now incorporate the notion of connected trajectories, finding a probability density function for quantum trajectories and their mostlikely paths. These most-likely paths describe routes with the highest probability density, taking into account that all of the points in the trajectory ensemble are connected via quantum-state update rules, e.g., in Eq. (2). As we have seen previously, the concurrence distribution exhibits a transition from a single-peak distribution to double-peak distributions, one at the upper bound concurrence and another near zero concurrence. Here, with the notion of trajectories, we also see that the two-qubit state starting in its initial state gradually collapses to three subspaces: the $|00\rangle$ subspace, the $|11\rangle$ subspace, and the odd subspace, as described by three most-likely routes.

Let us consider the five nontrivial elements of the twoqubit density matrix $\left\{x_{1}, x_{2}, x_{3}, x_{4}, x_{5}\right\}$ and treat each element as an independent variable. Each state trajectory is represented by a time series of these five elements, which corresponds to one realization of the measurement readout $\left\{v_{t}\right\}=\left\{v_{0}, v_{\delta t}, v_{2 \delta t}, \ldots, v_{t}\right\}$, where we define $v_{t}=$ $(f / \delta t) \int_{t}^{t+\delta t} \tilde{V}\left(t^{\prime}\right) \mathrm{d} t^{\prime}-v_{0}$ as an instantaneous readout at time $t$ with an integration time $\delta t$. Since the readout $v_{t}$ 's are assumed to be Markovian and only depend on the qubit states right before the measurement, a joint probability density function for the readout realization is given by

$$
\mathcal{P}\left(\left\{v_{t}\right\}\right)=\prod_{t^{\prime}=0}^{t}\left\{\sum_{k=1}^{4} x_{k, t^{\prime}} p\left(v_{t^{\prime}} \mid k\right)\right\},
$$

a product of probability distributions of $v_{t^{\prime}}$ from $t^{\prime}=0$ to $t^{\prime}=t$ with a time step $\delta t$. The probability density function for an instantaneous readout is given by $p\left(v_{t} \mid i\right)=$ $\sqrt{\delta t / \pi s} \exp \left\{-\left(v_{t}-\delta v_{i}\right)^{2} \delta t / s\right\}$ for $i=1,2,3,4$.

To derive the most-likely path for these two-qubit trajectories, we use the Bayesian update equation for the two-qubit state Eq. (2), adapted to a state update every time step $\delta t$, and then we maximize the joint probability density Eq. (12), constraining the state update equations. Following the most-likely path analysis for quantum states under continuous measurement [36,37] and introducing Lagrange multipliers $\left\{p_{1}, p_{2}, p_{3}, p_{4}, p_{5}\right\}$ for the constraints, we obtain differential equations for an optimal path in the qubit-state space,

$$
\begin{aligned}
\partial_{t} x_{i}= & +\frac{x_{i}}{s} \sum_{k=1}^{4} x_{k}\left\{2 v_{t}\left(\delta v_{i}-\delta v_{k}\right)-\delta v_{i}^{2}+\delta v_{k}^{2}\right\}, \\
\partial_{t} x_{5}= & -\gamma x_{5}+\frac{x_{5}}{s}\left\{v_{t}\left(\delta v_{2}+\delta v_{3}\right)-\frac{\left(\delta v_{2}^{2}+\delta v_{3}^{3}\right)}{2}\right. \\
& \left.+\sum_{k=1}^{4} x_{k}\left(\delta v_{k}^{2}-2 v_{t} \delta v_{k}\right)\right\},
\end{aligned}
$$

where $i=1, \ldots, 4$ for the first line and the variables $x_{k}$ are time-dependent functions. We note that $v_{t}$ in these equations behaves as a "smooth" optimal readout, which is a function of the qubit density matrices and their Lagrange multipliers, determining an optimal path from an initial state to its final state [36]. An optimal path is a solution of ten differential equations: five for the qubit-state variables, Eq. (13), another five for Lagrange multipliers, and the optimal readout as a function of both sets of variables (see Appendix A). These equations describe most-likely paths for a measurement with any values of $\delta v_{1, \ldots, 4}$ and can also be generalized to include the effect of external drives on the two-qubit state. However, in the absence of an external drive, these can be simplified, as we will see in the next section.

\section{A. Most-likely paths for joint measurement of transmon qubits}

In this work, where the transmon qubits only evolve with the influence of the measurement backaction, the optimal readout is found to be constant in time (see Appendix A). We can therefore bypass solving the full set of differential equations [49] and only compute the qubit evolution in Eq. (13) with constant $v_{t}$, looking for measurement results with maximum likelihood density. To estimate the likelihood density of a two-qubit trajectory, we evaluate a logarithm of the probability density function Eq. (12) approximated to first order in $\delta t$ [36] to obtain

$\log \mathcal{P}\left(\left\{v_{t}\right\}\right) \approx \mathcal{S}_{0}-\int_{0}^{t} \mathrm{~d} t^{\prime}\left\{\frac{1}{s} \sum_{k=1}^{4}\left(v_{t^{\prime}}-\delta v_{k}\right)^{2} x_{k, t^{\prime}}\right\}$, 
where $\mathcal{S}_{0}$ represents a state-independent part of the joint probability density function $\mathcal{P}$. We show in the insets of Figs. 3(a)-3(c) examples of the approximated loglikelihood density as functions of optimal readout $v_{t}$, for three different measurement strengths. In the case of $\tau_{m} \approx 2.10 \mu \mathrm{s}$, there is only one maximum likelihood value located at $v_{t} \approx \delta v_{2,3}$, which gives the most-likely path with high concurrence, shown as a solid curve in Fig. 3(a); whereas, in the stronger measurement cases, Figs. 3(b) and (c), the approximated log-likelihood density has three local maxima: the middle ones, $v_{t} \approx \delta v_{2,3}$, corresponding to most-likely paths collapsed to entangled states (high-concurrence branches); and the sided peaks, $v_{t} \approx \delta v_{1}$ and $v_{t} \approx \delta v_{4}$, corresponding to two branches of most-likely paths collapsed to $|00\rangle$ and $|11\rangle$ states (low-concurrence branches), respectively. We note that this technique of finding multiple most-likely paths is not under final-state constraints as in Refs. [35,36].

We choose to compare the theoretical most-likely paths with high-trajectory density paths extracted from the experimental data, using the trace distance method. We collect an ensemble of $10^{4}$ transmon trajectories and then compute average trace distances between any two trajectories (e.g., $\rho_{a}$ and $\rho_{b}$ ),
$D_{a, b}=\frac{\delta t}{2 T} \sum_{t^{\prime}=0}^{t} \operatorname{Tr}\left(\sqrt{\left(\rho_{a}\left(t^{\prime}\right)-\rho_{b}\left(t^{\prime}\right)\right)^{\dagger}\left(\rho_{a}\left(t^{\prime}\right)-\rho_{b}\left(t^{\prime}\right)\right)}\right)$

for all possible pairs. The goal is to pick the first few trajectories with minimum total distance to other trajectories in the ensemble and average them to get an estimate of the experimental most-likely paths. For this particular set of data, we choose about $10^{2}$ highly likely trajectories to get a smooth estimate of the most-likely paths. However, for the case in which there exist multiple (e.g., three) most-likely paths, we first divide the ensemble into subensembles according to their trace distance and then apply the minimum total distance procedure to the trajectories in each subensemble separately. In Figs. 3(a)-3(c), we show the concurrence of the experimental most-likely paths as data points.

Ideally, the theoretical most-likely paths predicted from the log-likelihood density, Eq. (14), for each set of measurement strengths using the initial state $\left\{x_{1}^{0}, \ldots, x_{5}^{0}\right\}=$ $\{1 / 4, \ldots, 1 / 4\}$ would be good enough to compare with the experimental data. However, in the experiment, after the initial state has been prepared, the cavities take some time
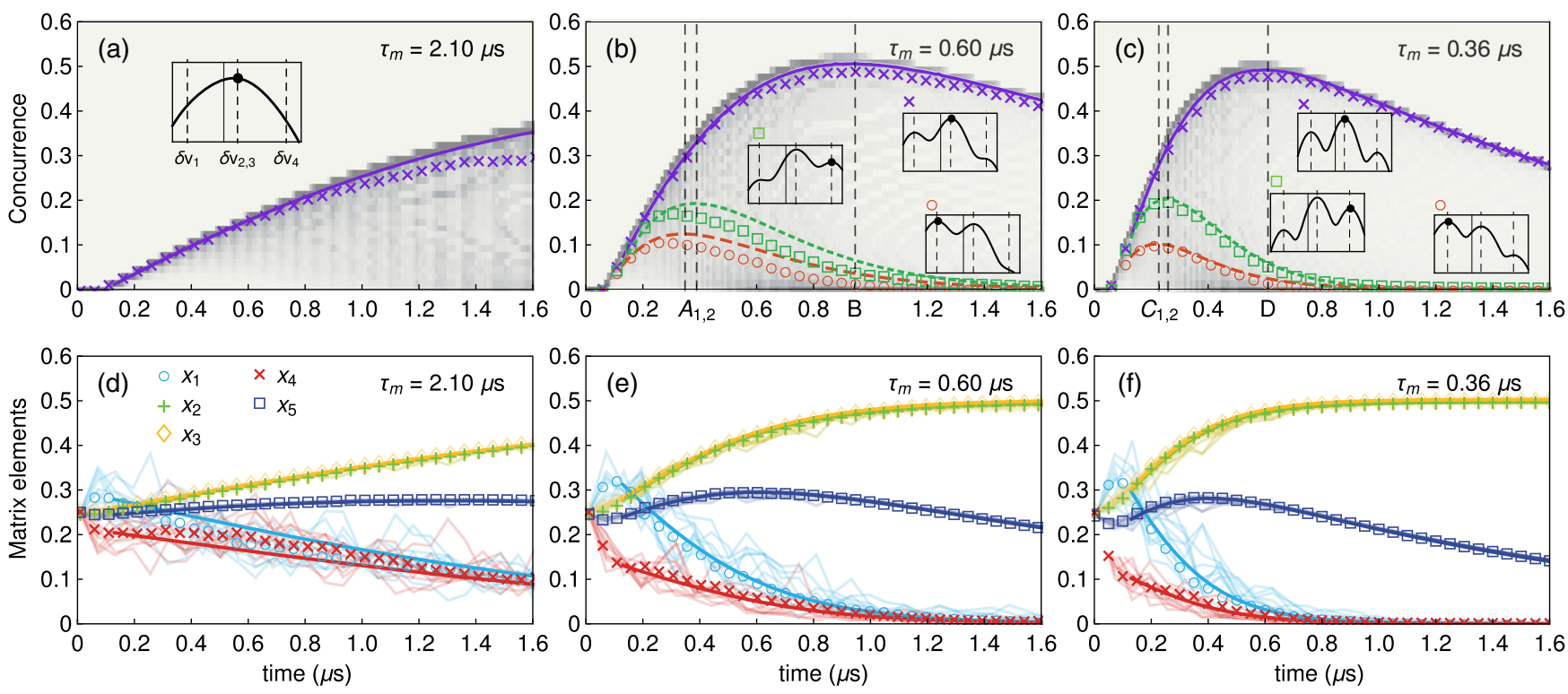

FIG. 3. The most-likely paths from the theoretical prediction and the experimental data. The first, second, and third columns are from three different data sets, measuring the same qubits and cavities with three different measurement-readout powers (indicated by the characteristic measurement time): $\tau_{m}=2.10 \mu \mathrm{s}, 0.60 \mu \mathrm{s}$, and $0.36 \mu \mathrm{s}$, respectively. Panels (a)-(c) show the concurrence of the multiple most-likely paths: the path with high concurrence (solid purple line), and the two paths with low concurrence projecting onto $|00\rangle$ (dashed orange line) and $|11\rangle$ (dotted green line) subspaces. The vertical lines (labeled as $A_{1,2}, B, C_{1,2}, D$ ) represent the times at which the concurrence is maximum for each of these paths. The insets show the log-likelihood density as functions of optimal measurement readouts, and the grey-scale histograms in the background show experimental concurrence histograms similar to the one in Fig. 2(c). Panels (d)-(f) present the evolution of the density matrix elements of the high-concurrence most-likely paths: The solid curves indicate theoretical solutions, whereas the data points indicate experimental most-likely paths postselected with the most entangled state at the final time $T=1.6 \mu$ s. Examples of the postselected trajectories are shown as fluctuating curves. 
to reach their steady-state condition, making the parameters $\delta v_{1}, \ldots, \delta v_{4}$ unstable during the first $\sim 0.13 \mu$ s time. Therefore, we need to let the initial qubit state evolve and then find new "initial" states at time $t=0.13 \mu \mathrm{s}$ for the theoretical most-likely path calculation. We use the experimental most-likely states at $t=0.13 \mu \mathrm{s}$ (one for each branch) as the initial states in calculating the log-likelihood density functions (the insets), which then leads to an excellent prediction of the most-likely paths and their concurrence for the rest of the evolution.

We also note that the two unequal low-concurrence branches in Figs. 3(b) and 3(c) happen because the population of the states drifts more toward the ground state $|00\rangle$ during the transient time, as a result of the qubit relaxation during the measurement. Moreover, in Figs. 3(d)-3(f), we present the evolution of matrix elements of the high-concurrence most-likely paths, showing a good agreement between the theoretical most-likely paths and experimental most-likely paths postselected with the most entangled state at the final time $T=1.6 \mu \mathrm{s}$.

\section{B. Most-likely paths for perfectly symmetric half-parity measurement}

We are also interested in finding analytic solutions for the most-likely paths for the symmetric case: $\delta v_{2}=\delta v_{3}=0$ and $-\delta v_{1}=\delta v_{4}=\delta v$. The differential equations (13) for the qubit state simplify to

$$
\begin{gathered}
\partial_{t} x_{p}=-b x_{p}-a x_{m}+a x_{p} x_{m}+b x_{p}^{2}, \\
\partial_{t} x_{m}=-b x_{m}-a x_{p}+b x_{m} x_{p}+a x_{m}^{2}, \\
\partial_{t} x_{2,3}=+x_{2,3}\left(a x_{m}+b x_{p}\right), \\
\partial_{t} x_{5}=-\gamma x_{5}+x_{5}\left(a x_{m}+b x_{p}\right),
\end{gathered}
$$

where we have defined new variables $x_{p} \equiv x_{1}+x_{4}$ and $x_{m} \equiv x_{1}-x_{4}$, and constant parameters $a=v_{t} \delta v / s$ and $b=\delta v^{2} / 4 s$. The first two equations can be solved independently from the rest. For the case $v_{t}=\delta v_{2,3}=0$, which corresponds to the most-likely odd-parity result, we obtain an analytic solution for the high-concurrence branch of the most-likely path,

$$
\begin{gathered}
x_{1,4}(t) \propto x_{1,4}^{0} \exp \left(-\delta v^{2} t / 4 s\right), \\
x_{2,3}(t) \propto x_{2,3}^{0}, \\
x_{5}(t) \propto x_{5}^{0} \exp (-\gamma t),
\end{gathered}
$$

where the proportionality factor is the inverse of a normalized factor $\mathcal{N}=\left(1-x_{1}^{0}-x_{4}^{0}\right)+\left(x_{1}^{0}+x_{4}^{0}\right) \exp \left(-\delta v^{2} t / 4 s\right)$. The concurrence of this path exactly coincides with the upper concurrence bound derived in Eq. (11), which is not surprising because the distribution of concurrence shows sharp peaks along the concurrence bound. We note that the solutions for the low-concurrence branch (for $v_{t}=\delta v$ and $v_{t}=-\delta v$ ) can be found numerically.

In the above analysis, for the half-parity case, a simple analytic solution for the most-likely path for arbitrary values of $v_{t}$ was not forthcoming. However, if we further simplify the problem by considering a parity meter [6], then we can solve the equations of motion, Eqs. (13) and their conjugate equations, exactly. A parity meter has the same detector outputs for the even- and odd-parity subspaces, but the detector can distinguish between the subspaces. More detail of the calculation is presented in Appendix C.

\section{Distribution of time to maximum concurrence}

In the process of the entanglement generation, there are interesting quantities to investigate, such as the maximum concurrence each individual trajectory can reach and the time it takes to reach the highest value. We previously showed that qubit trajectories branch out to high- and lowconcurrence subspaces. Therefore, one would expect that there are two most-likely times for the qubit trajectories to reach their maximum concurrences (or their most entangled states).

In Fig. 4, we show the normalized histograms of time for transmon qubit trajectories to reach their maximum concurrence. The histograms for the $\tau_{m}=0.36 \mu \mathrm{s}$ and $0.60 \mu \mathrm{s}$ measurement cases explicitly show double peaks, which agree with the branching of concurrence and the mostlikely qubit paths in Figs. 3(b) and 3(c). The times at which these peaks are located can be theoretically predicted from

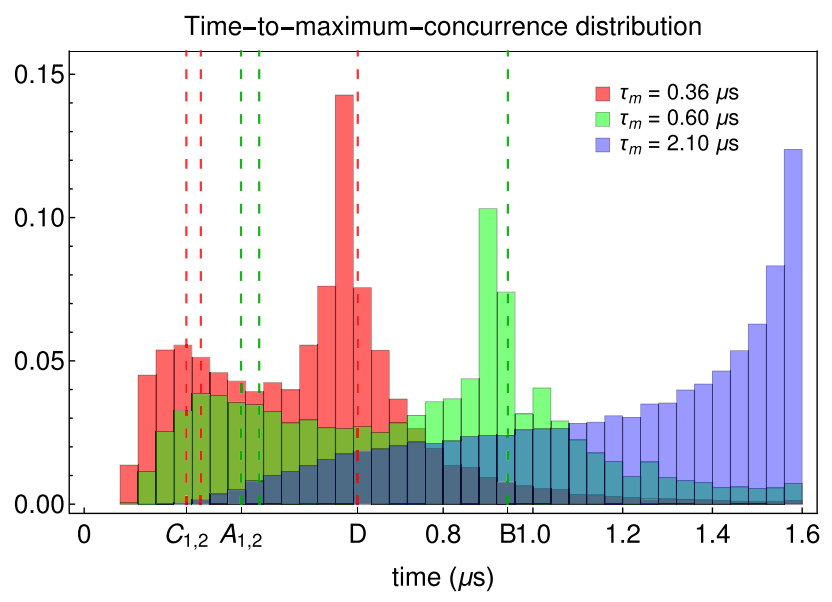

FIG. 4. Histograms of the time to maximum concurrence for individual trajectories for three measurement strengths indicated by the values of $\tau_{m}$. For the two cases with strong readout powers (shown in red and green histograms), there exists two peaks corresponding to two most-likely times to reach their most entangled states. The theoretical predictions of these times are shown as vertical dashed lines labeled as $A_{1,2}, B, C_{1,2}, D$ [same as in Figs. 3(a)-3(c)]. 
the time to maximum concurrence of the solutions of the most-likely paths. As shown by the vertical dashed lines in Figs. 3(b), 3(c), and 4, $A_{1,2}$ and $B$ are the two most-likely times to reach maximum concurrence (for low- and highconcurrence branches, respectively) for $\tau_{m}=0.60 \mu \mathrm{s}$, and $C_{1,2}$ and $D$ are the same but for the case with $\tau_{m}=0.36 \mu \mathrm{s}$. The agreement between the theoretical prediction of the peaks and the peaks of the histograms are as good as the agreement of the theory-experiment most-likely paths in Figs. 3(b) and 3(c). We note that for the weak measurement regime, $\tau_{m}=2.10 \mu \mathrm{s}$, the bifurcation has not occurred yet during the measurement time $T=1.6 \mu \mathrm{s}$. One would expect to see a branching effect when the total measurement time is long enough.

\section{CONCLUSION}

We have investigated the process of entanglement generation between two spatially separated superconducting transmon qubits and their statistical properties. The entanglement of the two qubits is created as a result of the halfparity dispersive measurement via the microwave pulses sequentially interacting with both qubits. The strength of the joint measurement is arbitrary, and we have studied three different values of the measurement strength. We examined the concurrence of individual trajectories and theoretically calculated its distribution from the quantum Bayesian approach, gradually projecting the two-qubit states to entangled states with high concurrence and to separable states with zero concurrence.

The most-likely path analysis was also carried out, predicting the most probable paths for the qubit trajectories. We found that in the two-qubit-state space, there are three likely paths conforming to the three branches projecting to the $|00\rangle$ subspace, the $|11\rangle$ subspace, and the odd $(|01\rangle$, $|10\rangle)$ subspaces; the first two correspond to the lower branches of the concurrence bifurcation, and the last corresponds to the high-concurrence branch. These theoretical most-likely paths show good agreement with the experimental most-likely ones extracted from the transmon trajectory data (for three independent data sets) based on the trace distance between trajectories in two-qubit-state space. Moreover, we have presented the distributions of the time to the maximum concurrence for individual trajectories. The most-likely path analysis was shown to be useful in predicting the peaks of these time distributions.

We conclude that the accurate tracking of quantum trajectories of a jointly measurement qubit system is possible and that the physics of the entanglement creation statistics is well described by a quantum trajectory theoretical approach. The theoretical most-likely paths to entanglement and concurrence distribution match the experiment excellently. This work shows the way to use this process as a control mechanism to entangle remote systems for quantum-information-processing purposes. In future work, similar questions can be posed about the full-parity measurement, and we have made some predictions about that case in this work.

\section{ACKNOWLEDGMENTS}

This work was supported by US Army Research Office Grants No. W911NF-15-1-0496 and No. W911NF-13-10402, by National Science Foundation Grant No. DMR1506081, by John Templeton Foundation Grant ID 58558, and by the Development and Promotion of Science and Technology Talents Project Thailand. M. E. K. S. acknowledges support from the Fannie and John Hertz Foundation.

\section{APPENDIX A: OPTIMIZED PATHS (MOST- LIKELY PATHS) WITH PRESELECTED OR POSTSELECTED STATES}

We follow the outline in Refs. [36,37] for the stochastic path-integral formalism and the action principle for continuous quantum measurement. A joint probability density function of quantum states and measurement readouts, from time $t^{\prime}=0$ to $t^{\prime}=t$, is given by a path integral

$$
\begin{gathered}
\mathcal{P}\left(\left\{\boldsymbol{x}_{t}\right\},\left\{v_{t}\right\} \mid \boldsymbol{x}_{0}\right)=\mathcal{N} \int \mathcal{D} \boldsymbol{p}(t) \exp \mathcal{S}, \\
\mathcal{S}=-\int_{0}^{t} \mathrm{~d} t^{\prime}\left\{\frac{1}{s} \sum_{i=1}^{4}\left(v_{t^{\prime}}-\delta v_{i}\right)^{2} x_{i}+\sum_{j=1}^{5} p_{j}\left(\partial_{t^{\prime}} x_{j}-\mathcal{F}_{j}\right)\right\},
\end{gathered}
$$

where $\left\{\boldsymbol{x}_{t}\right\}=\left\{x_{j, t^{\prime}}\right\}$ denotes a set of quantum-state matrix elements at all times (with $j=1,2, \ldots, 5$, and $t^{\prime}$ runs from time $t^{\prime}=0$ to $t^{\prime}=t$ ), and $\mathcal{D} \boldsymbol{p}(t)$ denotes the path-integral measure for the conjugate variables (or Lagrange multipliers as mentioned in the main text). The action of the path integral $\mathcal{S}$ [37] is given in terms of the two-qubit density matrix variables $x_{j}$ and their conjugates $p_{j}$ for $j=1,2, \ldots, 5$, which are implicitly functions of time $t^{\prime}$. The first sum in the time integral of $\mathcal{S}$ is the logarithm of the joint probability density function of the measurement readout $\mathcal{P}\left(\left\{v_{t}\right\}\right)$, Eq. (12), truncated to first order in $\delta t$ (taking a time continuum limit), and the functional $\mathcal{F}_{j}$ is the right-hand side of Eq. (13).

The joint probability density function in a path-integral representation, Eq. (A2), is written with a measure $\mathcal{D} \boldsymbol{x}(t) \mathcal{D} v(t) \equiv \lim _{\delta t \rightarrow 0} \prod_{t^{\prime}=0}^{t} \mathrm{~d} x_{1, t^{\prime}} \mathrm{d} x_{2, t^{\prime}} \cdots \mathrm{d} x_{5, t^{\prime}} \mathrm{d} v_{t^{\prime}}, \quad$ considering the density matrix elements and the measurement readout as random variables. The probability density function multiplied by the measure gives a probability that is vanishingly small but invariant under changes of measure. However, as in usual probability theory, one can ask about "peaks" or the most-likely values of an associated probability density function. The most-likely paths for the quantum trajectories starting from an initial state and 
ending at a final state after some time $t$ can be found by optimizing the action of the stochastic path integral (A2).

By extremizing the action Eq. (A2) over all variables $x_{i}$, $p_{i}, v_{t}$, we get a set of ten ordinary differential equations (ODEs) for the optimized path, and one equation for optimal measurement readout. The set of ODEs includes five differential equations of the two-qubit variables $x_{i}$, as shown in Eq. (13), and five equations for the conjugate variables $p_{i}$,

$$
\begin{gathered}
\partial_{t} p_{i}=\sum_{j=1}^{4} x_{j} A_{i j}+x_{5} p_{5} B_{i}+C_{i} \quad \text { for } i=1,2,3,4, \\
\partial_{t} p_{5}=+\gamma_{5} p_{5}+p_{5} \sum_{j=1}^{4} x_{j} B_{j},
\end{gathered}
$$

where

$$
\begin{aligned}
A_{i j} & =-\left(p_{i}-p_{j}\right)\left(2 v_{t}-\delta v_{i}-\delta v_{j}\right)\left(\delta v_{i}-\delta v_{j}\right), \\
B_{i} & =\frac{1}{2 s}\left\{v_{t}\left(4 \delta v_{i}-2 \delta v_{2}-2 \delta v_{3}\right)-\left(2 \delta v_{i}^{2}-\delta v_{2}^{2}-\delta v_{3}^{2}\right)\right\}, \\
C_{i} & =\frac{1}{2 s}\left(v_{t}-\delta v_{i}\right)^{2} .
\end{aligned}
$$

The optimal readout is given as a function of the two-qubit variables and the conjugate variables,

$$
\begin{aligned}
v_{t}= & \sum_{i=1}^{4} \sum_{j=1}^{4}\left\{p_{i} x_{i} x_{j}\left(\delta v_{i}-\delta v_{j}\right)+x_{i} \delta v_{i}\right\} \\
& +\frac{p_{5} x_{5}}{2} \sum_{i=1}^{4}\left(\delta v_{2}+\delta v_{3}-2 x_{i} \delta v_{i}\right),
\end{aligned}
$$

connecting the ODEs for the qubit variables and the conjugate variables. We note that by taking a time derivative of the function in Eq. (A4) and substituting both $\partial_{t} x_{i}$ and $\partial_{t} p_{j}$ with the ODEs above, we find that $\partial_{t} v_{t}=0$, therefore implying that an optimal readout is a constant in time.

From the joint probability density function (A1), we can consider integrating out the measurement-readout variables to get a probability density function just in the quantumstate variables, i.e., $\mathcal{P}\left(\left\{\boldsymbol{x}_{t}\right\} \mid \boldsymbol{x}_{0}\right)=\int \mathcal{D} v(t) \mathcal{P}\left(\left\{\boldsymbol{x}_{t}\right\}\right.$, $\left.\left\{v_{t}\right\} \mid \boldsymbol{x}_{0}\right)$. Since the action (A2) is bilinear in $v_{t}$, its Gaussian-functional integral gives exactly the same result (up to a constant normalization factor) as extremizing the action over the readout and substituting the optimal solution (A4) to the action. By extremizing the rest of the action over the quantum-state variables and their conjugates, we get the same set of ten differential equations [Eqs. (13) and (A3)] with the substitution of the optimal readout (A4). The solutions to these equations can now be interpreted as an optimal path in the quantum-state space only. This similar definition of the optimal path is also used in classical stochastic processes, for example, by the variation of the Martin-Siggia-Rose action formalism [56,57].

\section{APPENDIX B: TRANSFORMATION OF THE MOST-LIKELY PATH UNDER CHANGE OF MEASURE}

We further investigate the transformations of the optimal paths under change of measure. In probability theory, given a probability density function of a continuous random variable $V$, one can consider a change of variable $V \rightarrow \zeta=g(V)$, where $\zeta$ is a new random variable. The total probability contained in the differential region should be invariant, i.e., $p_{V}(V) \mathrm{d} V=p_{\zeta}(\zeta) \mathrm{d} \zeta$, so the new probability density function can be reformulated as $p_{\zeta}(\zeta)=p_{V}\left(g^{-1}(\zeta)\right)\left|[d /(d \zeta)] g^{-1}(\zeta)\right|$, assuming that $g$ is monotonic. If there exists an optimal value $V_{\text {opt }}$, obtained by solving $\left.[d /(d V)] p_{V}(V)\right|_{V_{\text {opt }}}=0$, and there similarly exists an optimal value in the $\zeta$ variable, one can show that $\zeta_{\text {opt }}$ does not need to be the same as $g\left(V_{\text {opt }}\right)$. Thus, a question regarding an optimal (most-likely) value is dependent on the random variables one is interested in; that is, it is measure dependent. We also note that for a special case when $g(V)$ is a linear function, the optimal values in the two variables coincide, $\zeta_{\mathrm{opt}}=g_{\text {linear }}\left(V_{\mathrm{opt}}\right)$.

We stress that, even though the joint optimization of the quantum states and the measurement readouts (A1) can give a different solution under a change of the readout variables, we can avoid this change of measure by integrating out all the readout variables and only considering the optimal path in the quantum-state space, as discussed at the end of the previous appendix. For the rescaled shifted homodyne signal as a readout variable, the joint optimization happens to coincide with the quantumstate optimization after integrating out the readout variables. This way we have thus proven that our quantum-state optimal path is independent of the readout measure, while the optimal readout itself $v_{t}$ is a measure-dependent quantity in general.

\section{APPENDIX C: ANALYTIC SOLUTION IN THE CASE OF A PARITY METER}

For the full-parity measurement case, we assume $\delta v_{1}=\delta v_{4}=d v$, while $\delta v_{2}=\delta v_{3}=0$. With this special case, we find the equations of motion

$$
\begin{aligned}
& \partial_{t} x_{1}=+\lambda x_{1} x_{o}, \\
& \partial_{t} x_{2}=-\lambda x_{2} x_{e}, \\
& \partial_{t} x_{3}=-\lambda x_{3} x_{e}, \\
& \partial_{t} x_{4}=+\lambda x_{4} x_{o},
\end{aligned}
$$




$$
\partial_{t} x_{5}=-\gamma x_{5}-\lambda x_{5} x_{e}
$$

where we define $\lambda=2 v_{t} \delta v / s-\delta v^{2} / s$, and $x_{e}=x_{1}+x_{4}=$ $\rho_{00,00}+\rho_{11,11}$ is the probability of being in the even-parity subspace, while $x_{o}=x_{2}+x_{3}=\rho_{01,01}+\rho_{10,10}$ is the probability of being in the odd-parity subspace.

Taking the sum of $\partial_{t} x_{1}$ and $\partial_{t} x_{4}$, we can derive an equation for $x_{o}$ alone since $x_{e}+x_{o}=1$,

$$
\dot{x}_{o}=-\dot{x}_{e}=-\lambda\left(1-x_{o}\right) x_{o} .
$$

Integrating this equation gives the solution

$$
x_{o}(t)=\frac{x_{o}^{0} e^{-\lambda t}}{1-x_{o}^{0}\left(1-e^{-\lambda t}\right)},
$$

where $x_{o}^{0}$ is the initial condition for $x_{2}+x_{3}$. Similarly, we find for the even probability,

$$
x_{e}(t)=\frac{x_{e}^{0}}{1-x_{o}^{0}\left(1-e^{-\lambda t}\right)},
$$

where $x_{e}^{0}$ is the initial condition for $x_{1}+x_{4}$.

Notice that the value of $\lambda$ may be found completely from these results. The parity probability of the initial preselected and final postselected states will fix the value of $\lambda$. If the integrated signal gives a positive answer larger than $d v / 2, \lambda$ will be positive, tending to collapse the state into the even-parity subspace. Conversely, if the integrated signal is less than $d v / 2$, then $\lambda$ will be negative, tending to collapse the system into the odd-parity subspace.

From these solutions, we may find the other density matrix solutions. For example, Eq. (C1a) may be rewritten as $\partial_{t} \ln x_{1}=\lambda x_{o}$, which can be integrated to get $x_{1}(t)=x_{1}^{0} \exp \left\{\lambda \int_{0}^{t} \mathrm{~d} t^{\prime} x_{o}\left(t^{\prime}\right)\right\}$. We simply apply this method to the rest of Eqs. (C1) and obtain the solutions, the most-likely path of the two-qubit problem,

$$
\begin{aligned}
& x_{1}(t)=\frac{x_{1}^{0}}{1-\left(x_{2}^{0}+x_{3}^{0}\right)\left(1-e^{-\lambda t}\right)}, \\
& x_{2}(t)=\frac{x_{2}^{0} e^{-\lambda t}}{1-\left(x_{2}^{0}+x_{3}^{0}\right)\left(1-e^{-\lambda t}\right)}, \\
& x_{3}(t)=\frac{x_{3}^{0} e^{-\lambda t}}{1-\left(x_{2}^{0}+x_{3}^{0}\right)\left(1-e^{-\lambda t}\right)}, \\
& x_{4}(t)=\frac{x_{4}^{0}}{1-\left(x_{2}^{0}+x_{3}^{0}\right)\left(1-e^{-\lambda t}\right)}, \\
& x_{5}(t)=\frac{x_{5}^{0} e^{-(\gamma+\lambda) t}}{1-\left(x_{2}^{0}+x_{3}^{0}\right)\left(1-e^{-\lambda t}\right)},
\end{aligned}
$$

where the value of $\lambda$ can be fixed once we have specified the initial and final values of the density matrix, $x_{1}^{0}, x_{2}^{0}, \ldots$, $x_{5}^{0}, x_{1, f}, x_{2, f}, \ldots, x_{5, f}$.

Finally, we may find the concurrence of the most-likely path by calculating $\mathcal{C}=2 \max \left\{0, x_{5}-\sqrt{x_{1} x_{4}}\right\}$. We find the result

$$
\mathcal{C}(t)=2 \max \left\{0, \frac{x_{5}^{0}\left|e^{-(\gamma+\lambda) t}\right|-\sqrt{x_{1}^{0} x_{4}^{0}}}{\left|1-\left(x_{2}^{0}+x_{3}^{0}\right)\left(1-e^{-\lambda t}\right)\right|}\right\}
$$

In order to cross the entanglement border [6] from an unentangled state, $\lambda$ must take on a negative value to enhance the first term over the second, taking the system into the odd-parity subspace.

The entanglement border will be crossed when the concurrence is zero or when the condition

$$
\gamma+\lambda=(1 / t) \ln \left(\frac{x_{5}^{0}}{\sqrt{\mid x_{1}^{0} x_{4}^{0 \mid}}}\right)
$$

is satisfied.

[1] C. K. Hong, Z. Y. Ou, and L. Mandel, Measurement of Subpicosecond Time Intervals between Two Photons by Interference, Phys. Rev. Lett. 59, 2044 (1987).

[2] A. N. Korotkov, Continuous Measurement of Entangled Qubits, Phys. Rev. A 65, 052304 (2002).

[3] R. Ruskov and A. N. Korotkov, Entanglement of Solid-State Qubits by Measurement, Phys. Rev. B 67, 241305 (2003).

[4] W. Mao, D. V. Averin, R. Ruskov, and A. N. Korotkov, Mesoscopic Quadratic Quantum Measurements, Phys. Rev. Lett. 93, 056803 (2004).

[5] B. Trauzettel, A. N. Jordan, C. W. J. Beenakker, and M. Büttiker, Parity Meter for Charge Qubits: An Efficient Quantum Entangler, Phys. Rev. B 73, 235331 (2006).

[6] N.S. Williams and A. N. Jordan, Entanglement Genesis under Continuous Parity Measurement, Phys. Rev. A 78, 062322 (2008).

[7] S. G. Hofer, D. V. Vasilyev, M. Aspelmeyer, and K. Hammerer, Time-Continuous Bell Measurements, Phys. Rev. Lett. 111, 170404 (2013).

[8] C. W. Chou, H. de Riedmatten, D. Felinto, S. V. Polyakov, S. J. van Enk, and H. J. Kimble, Measurement-Induced Entanglement for Excitation Stored in Remote Atomic Ensembles, Nature (London) 438, 828 (2005).

[9] D. L. Moehring, P Maunz, S Olmschenk, K. C. Younge, D. N. Matsukevich, L.-M. Duan, and C. Monroe, Entanglement of Single-Atom Quantum Bits at a Distance, Nature (London) 449, 68 (2007).

[10] H. Bernien, B. Hensen, W. Pfaff, G. Koolstra, M. S. Blok, L. Robledo, T. H. Taminiau, M. Markham, D. J. Twitchen, L. Childress, and R. Hanson, Heralded Entanglement between Solid-State Qubits Separated by Three Metres, Nature (London) 497, 86 (2013).

[11] B. Hensen, H. Bernien, A. E. Dréau, A. Reiserer, N. Kalb, M. S. Blok, J. Ruitenberg, R. F. L. Vermeulen, R. N. 
Schouten, C. Abellán et al., Loophole-Free Bell Inequality Violation Using Electron Spins Separated by 1.3 Kilometres, Nature (London) 526, 682 (2015).

[12] N. Roch, M. E. Schwartz, F. Motzoi, C. Macklin, R. Vijay, A. W. Eddins, A. N. Korotkov, K. B. Whaley, M. Sarovar, and I. Siddiqi, Observation of Measurement-Induced Entanglement and Quantum Trajectories of Remote Superconducting Qubits, Phys. Rev. Lett. 112, 170501 (2014).

[13] M. H. Devoret and R.J. Schoelkopf, Superconducting Circuits for Quantum Information: An Outlook, Science 339, 1169 (2013).

[14] K. W. Murch, R. Vijay, and I. Siddiqi, Weak Measurement and Feedback in Superconducting Quantum Circuits, in Superconducting Devices in Quantum Optics, edited by Robert H. Hadfield and Göran Johansson (Springer International Publishing, Switzerland, 2016), pp. 163-185.

[15] J. Ghosh, A. Galiautdinov, Z. Zhou, A. N. Korotkov, J. M. Martinis, and M. R. Geller, High-Fidelity Controlled- $\sigma^{Z}$ Gate for Resonator-Based Superconducting Quantum Computers, Phys. Rev. A 87, 022309 (2013).

[16] A. Fedorov, L. Steffen, M. Baur, M. P. Da Silva, and A. Wallraff, Implementation of a Toffoli Gate with Superconducting Circuits, Nature (London) 481, 170 (2011).

[17] M. Ansmann, H. Wang, R. C. Bialczak, M. Hofheinz, E. Lucero, M. Neeley, A. D O'Connell, D. Sank, M. Weides, J. Wenner et al., Violation of Bell's Inequality in Josephson Phase Qubits, Nature (London) 461, 504 (2009).

[18] R. C. Bialczak, M. Ansmann, M. Hofheinz, E. Lucero, M. Neeley, A. D. O'Connell, D. Sank, H. Wang, J. Wenner, M. Steffen et al., Quantum Process Tomography of a Universal Entangling Gate Implemented with Josephson Phase Qubits, Nat. Phys. 6, 409 (2010).

[19] B. Vlastakis, G. Kirchmair, Z. Leghtas, S. E. Nigg, L. Frunzio, S. M. Girvin, M. Mirrahimi, M. H. Devoret, and R. J. Schoelkopf, Deterministically Encoding Quantum Information Using 100-Photon Schrödinger Cat States, Science 342, 607 (2013).

[20] M. A. Castellanos-Beltran, K. D. Irwin, G. C. Hilton, L. R. Vale, and K. W. Lehnert, Amplification and Squeezing of Quantum Noise with a Tunable Josephson Metamaterial, Nat. Phys. 4, 929 (2008).

[21] M. Hatridge, R. Vijay, D. H. Slichter, John Clarke, and I. Siddiqi, Dispersive Magnetometry with a Quantum Limited SQUID Parametric Amplifier, Phys. Rev. B 83, 134501 (2011).

[22] N. Katz, M. Ansmann, R. C. Bialczak, E. Lucero, R. McDermott, M. Neeley, M. Steffen, E. M. Weig, A. N. Cleland, J. M. Martinis, and A. N. Korotkov, Coherent State Evolution in a Superconducting Qubit from PartialCollapse Measurement, Science 312, 1498 (2006).

[23] A. N. Korotkov and A. N. Jordan, Undoing a Weak Quantum Measurement of a Solid-State Qubit, Phys. Rev. Lett. 97, 166805 (2006).

[24] N. Katz, M. Neeley, M. Ansmann, R. C. Bialczak, M. Hofheinz, E. Lucero, A. O'Connell, H. Wang, A. N. Cleland, J. M. Martinis, and A. N. Korotkov, Reversal of the Weak Measurement of a Quantum State in a Superconducting Phase Qubit, Phys. Rev. Lett. 101, 200401 (2008).
[25] J. P. Groen, D. Ristè, L. Tornberg, J. Cramer, P. C. de Groot, T. Picot, G. Johansson, and L. DiCarlo, PartialMeasurement Backaction and Nonclassical Weak Values in a Superconducting Circuit, Phys. Rev. Lett. 111, 090506 (2013).

[26] P. Campagne-Ibarcq, L. Bretheau, E. Flurin, A. Auffèves, F. Mallet, and B. Huard, Observing Interferences between Past and Future Quantum States in Resonance Fluorescence, Phys. Rev. Lett. 112, 180402 (2014).

[27] N. S. Williams and A. N. Jordan, Weak Values and the Leggett-Garg Inequality in Solid-State Qubits, Phys. Rev. Lett. 100, 026804 (2008).

[28] H. M. Wiseman and G. J. Milburn, Quantum Measurement and Control (Cambridge University Press, Cambridge, England, 2010).

[29] R. Vijay, C. Macklin, D. H. Slichter, S. J. Weber, K. W. Murch, R. Naik, A. N. Korotkov, and I. Siddiqi, Stabilizing Rabi Oscillations in a Superconducting Qubit Using Quantum Feedback, Nature (London) 490, 77 (2012).

[30] G. de Lange, D. Ristè, M. J. Tiggelman, C. Eichler, L. Tornberg, G. Johansson, A. Wallraff, R. N. Schouten, and L. DiCarlo, Reversing Quantum Trajectories with Analog Feedback, Phys. Rev. Lett. 112, 080501 (2014).

[31] K. W. Murch, S. J. Weber, C. Macklin, and I. Siddiqi, Observing Single Quantum Trajectories of a Superconducting Quantum Bit, Nature (London) 502, 211 (2013).

[32] A. N. Jordan, Quantum Physics: Watching the Wavefunction Collapse, Nature (London) 502, 177 (2013).

[33] P. Campagne-Ibarcq, P. Six, L. Bretheau, A. Sarlette, M. Mirrahimi, P. Rouchon, and B. Huard, Observing Quantum State Diffusion by Heterodyne Detection of Fluorescence, Phys. Rev. X 6, 011002 (2016).

[34] M. Naghiloo, N. Foroozani, D. Tan, A. Jadbabaie, and K. W. Murch, Mapping Quantum State Dynamics in Spontaneous Emission, Nat. Commun. 7, 11527 (2016).

[35] S. J. Weber, A. Chantasri, J. Dressel, A. N. Jordan, K. W. Murch, and I. Siddiqi, Mapping the Optimal Route between Two Quantum States, Nature (London) 511, 570 (2014).

[36] A. Chantasri, J. Dressel, and A. N. Jordan, Action Principle for Continuous Quantum Measurement, Phys. Rev. A 88, 042110 (2013).

[37] A. Chantasri and A. N. Jordan, Stochastic Path-Integral Formalism for Continuous Quantum Measurement, Phys. Rev. A 92, 032125 (2015).

[38] A. N. Jordan, A. Chantasri, P. Rouchon, and B. Huard, Anatomy of Fluorescence: Quantum Trajectory Statistics from Continuously Measuring Spontaneous Emission, Quantum Stud. Math. Found. 3, 237 (2016).

[39] G. Haack, H. Förster, and M. Büttiker, Parity Detection and Entanglement with a Mach-Zehnder Interferometer, Phys. Rev. B 82, 155303 (2010).

[40] F. Helmer and F. Marquardt, Measurement-Based Synthesis of Multiqubit Entangled States in Superconducting Cavity QED, Phys. Rev. A 79, 052328 (2009).

[41] K. Lalumière, J. M. Gambetta, and A. Blais, Tunable Joint Measurements in the Dispersive Regime of Cavity QED, Phys. Rev. A 81, 040301 (2010).

[42] J. Wang, H. M. Wiseman, and G. J. Milburn, Dynamical Creation of Entanglement by Homodyne-Mediated Feedback, Phys. Rev. A 71, 042309 (2005). 
[43] C. Hill and J. Ralph, Weak Measurement and Control of Entanglement Generation, Phys. Rev. A 77, 014305 (2008).

[44] C. Meyer zu Rheda, G. Haack, and A. Romito, On-Demand Maximally Entangled States with a Parity Meter and Continuous Feedback, Phys. Rev. B 90, 155438 (2014).

[45] D. Riste, M. Dukalski, C. A. Watson, G. de Lange, M. J. Tiggelman, Ya. M. Blanter, K. W. Lehnert, R. N. Schouten, and L. DiCarlo, Deterministic Entanglement of Superconducting Qubits by Parity Measurement and Feedback, Nature (London) 502, 350 (2013).

[46] F. Motzoi, K. B. Whaley, and M. Sarovar, Continuous Joint Measurement and Entanglement of Qubits in Remote Cavities, Phys. Rev. A 92, 032308 (2015).

[47] L. Martin, F. Motzoi, H. Li, M. Sarovar, and K. B. Whaley, Deterministic Generation of Remote Entanglement with Active Quantum Feedback, Phys. Rev. A 92, 062321 (2015).

[48] J. Govenius, Y. Matsuzaki, I. G. Savenko, and M. Möttönen, Parity Measurement of Remote Qubits Using Dispersive Coupling and Photodetection, Phys. Rev. A 92, 042305 (2015).

[49] M. Silveri, E. Zalys-Geller, M. Hatridge, Z. Leghtas, M. H. Devoret, and S. M. Girvin, Theory of Remote Entanglement via Quantum-Limited Phase-Preserving Amplification, Phys. Rev. A 93, 062310 (2016).

[50] A. Narla, S. Shankar, M. Hatridge, Z. Leghtas, K. Sliwa, E. Zalys-Geller, S. Mundhada, W. Pfaff, L. Frunzio, R. Schoelkopf, and M. Devoret, Robust Concurrent Remote Entanglement between Two Superconducting Qubits, Phys. Rev. X 6, 031036 (2016).
[51] A. N. Jordan and A. N. Korotkov, Qubit Feedback and Control with Kicked Quantum Nondemolition Measurements: A Quantum Bayesian Analysis, Phys. Rev. B 74, 085307 (2006).

[52] Theoretically, if there is no single-qubit unitary rotation and the initial matrix elements are real values, all elements would stay real during the measurement process. However, in the experiment, the two cavities are not exactly at the same frequency and the qubits are not equally coupled to their respective cavities, so the qubits experience different ac-Stark shifts. The off-diagonal element acquires a deterministic (i.e., predictable) nonzero phase that increases linearly in time and, critically, does not affect the degree of entanglement in the system. We therefore study the magnitude of this element and neglect the phase.

[53] J. M. Chow, L. DiCarlo, J. M. Gambetta, A. Nunnenkamp, L. S. Bishop, L. Frunzio, M. H. Devoret, S. M. Girvin, and R. J. Schoelkopf, Detecting Highly Entangled States with a Joint Qubit Readout, Phys. Rev. A 81, 062325 (2010).

[54] W. K. Wootters, Entanglement of Formation of an Arbitrary State of Two Qubits, Phys. Rev. Lett. 80, 2245 (1998).

[55] L. Jakbczyk and A. Jamrz, Generation of Werner States and Preservation of Entanglement in a Noisy Environment, Phys. Lett. A 347, 180 (2005).

[56] P. C. Martin, E. D. Siggia, and H. A. Rose, Statistical Dynamics of Classical Systems, Phys. Rev. A 8, 423 (1973).

[57] A. Kamenev, Field Theory of Non-Equilibrium Systems (Cambridge University Press, Cambridge, England, 2011). 